\title{
Point Symmetric 2-Structures
}

\author{
Helmut Karzel, Jarosław Kosiorek and Andrzej Matraś
}

\author{
Dedicated to Heinrich Wefelscheid
}

\begin{abstract}
We show that every symmetric 2-structure $\left(P, \mathfrak{G}_{1}, \mathfrak{G}_{2}, \mathfrak{K}\right)$ of the class (III) [cf. Karzel $\mathrm{H}$ et al. (Result. Math., submitted)] is point symmetric, i.e. any two orthogonal chains $A, B \in \mathfrak{K}$ intersect in exactly one point and that any two points $a, b \in P$ have exactly one midpoint $m:=a * b$ (with $\widetilde{m}(a)=b$ where $\widetilde{m}$ is the unique symmetry in the point $m) . \widetilde{P}:=\{\widetilde{p} \mid p \in P\}$ is invariant, i.e. $\forall a, b \in P: \widetilde{a} \circ \widetilde{b} \circ \widetilde{a} \in \widetilde{P}$. Therefore the pair $(P, \widetilde{P})$ is an invariant regular involution set and the loop derivation in a point $o \in P$ gives a $\mathrm{K}$-loop $(P,+)$ uniquely 2-divisible.
\end{abstract}

Mathematics Subject Classification (2010). 51A99.

Keywords. Net, 2-structure, symmetric chain structure, midpoint.

\section{Introduction and Notations}

This paper is a continuation of our investigations on symmetric 2-structures [5]. This is a generalization of double symmetric 2-structures (cf. [4]) which are closely related with sharply 2 -transitive permutation groups $[1,6]$. We will use the same definitions and notations as in $[5]$ and $\Sigma:=\left(P, \mathfrak{G}_{1}, \mathfrak{G}_{2}, \mathfrak{K}\right)$ will denote a symmetric 2 -structure. We recall that for any point $p \in P$ and any $i \in\{1,2\}$ $[p]_{i}$ denotes the generator of $\mathfrak{G}_{i}$ passing through the point $p$ and that two points $a, b \in P$ are called parallel if there is an $i \in\{1,2\}$ such that $[a]_{i}=[b]_{i}$ and not parallel otherwise. With $P^{(2)}$ we denote the set of all pairs of not parallel points and then (by definition of a 2-structure) to any pair $(a, b) \in P^{(2)}$ there exists exactly one chain $K \in \mathfrak{K}$ - which we denote by $\overline{a, b}$ - with $a, b \in K$. We collect some properties of $\Sigma$ :

Theorem 1.1. $\forall A, B \in \mathfrak{K}: \widetilde{A}(B) \in \mathfrak{K}$ and $\widetilde{\widetilde{A}(B)}=\widetilde{A} \circ \widetilde{B} \circ \widetilde{A}$ hence $\widetilde{\mathfrak{K}}:=$ $\{\widetilde{K} \mid K \in \mathfrak{K}\}$ is invariant. 
In [5] we presented a classification of symmetric 2-structures based on the cardinality of the set $(p \perp K):=\{L \in \mathfrak{K} \mid p \in L \wedge L \perp K\}$ with $p \in K$ of all chains passing through the point $p$ and which are orthogonal to the chain $K$. The symmetric 2-structures split into the three classes (cf. [5, Theorem 3.10]):

(I) There is a pair $(p, K) \in P \times \mathfrak{K}$ with $p \in K$ and $|(p \perp K)|>1$.

(II) There is a pair $(p, K) \in P \times \mathfrak{K}$ with $p \in K$ and $(p \perp K)=\emptyset$.

(III) There is a pair $(p, K) \in P \times \mathfrak{K}$ with $p \in K$ and $|(p \perp K)|=1$.

An automorphism $\alpha$ of 2-structure $\left(P, \mathfrak{G}_{1}, \mathfrak{G}_{2}, \mathfrak{K}\right)$ is called point reflection if $\alpha$ is involutory, has exactly one fixpoint $p$ and $\alpha(B)=B$ for any chain or generator $B \in \mathfrak{B}:=\mathfrak{K} \cup \mathfrak{G}_{1} \cup \mathfrak{G}_{2}$ passing through $p$ (cf. [5, Definition 2.1.]).

In this paper we assume that each symmetric 2-structure $\left(P, \mathfrak{G}_{1}, \mathfrak{G}_{2}, \mathfrak{K}\right)$ is of class (III). This condition is equivalent to one of the following (cf. [5, Theorem. 3.13]):

1. For each pair $(x, X) \in P \times \mathfrak{K}$ with $x \in X:|(x \perp X)|=1$.

2. For every $x \in P$ there is exactly one reflection in the point $x$.

We denote the only chain of the set $(p \perp K)$ by the same symbol and here we can form $(p \perp \perp K):=(p \perp(p \perp K))$. By (2) to each point $p \in P$ there corresponds exactly one reflection $\tilde{p}$ in $p$. By [5, Theorem 3.4 and Corollary 3.5] we have the first statement of the following theorem:

Theorem 1.2. If $K \in \mathfrak{K}$ and $p \in P$ then:

1. $p \in K \Leftrightarrow \widetilde{p}(K)=K \Leftrightarrow \widetilde{K} \circ \widetilde{p}$ is involutory $\Leftrightarrow \exists_{1} L \in \mathfrak{K}: \widetilde{K} \circ \widetilde{p}=\widetilde{L}$.

2. If $p \in K$ and $L \in \mathfrak{K}$ with $\widetilde{K} \circ \widetilde{p}=\widetilde{L}$ then $L=(p \perp K)$.

3. If $p \notin K$ then $|(p \perp K)|=1$.

4. $\forall(a, b) \in P^{(2)}:(a \perp \overline{a, b}) \cap(b \perp \overline{a, b})=\emptyset$.

5. $\forall a, b \in P: \widetilde{\widetilde{a}(b)}=\widetilde{a} \circ \widetilde{b} \circ \widetilde{a}$ hence $\widetilde{P}:=\{\widetilde{p} \mid p \in P\}$ is invariant.

Proof. (2): Since $\widetilde{K} \circ \widetilde{L}=\widetilde{p} \neq i d, L \neq K$ and there is a $x \in L \backslash K$ hence $\widetilde{L}(x)=$ $x, \widetilde{K}(x) \neq x$. Moreover $\widetilde{L}(\widetilde{K}(x))=\widetilde{K}(\widetilde{L}(x))=\widetilde{K}(x)$ hence $L=\overline{x, \widetilde{K}(x)} \perp K$ and $\widetilde{L}(p)=\widetilde{K} \circ \widetilde{p}(p)=\widetilde{K}(p)=p$, i.e. $p \in L$ and so $L=(p \perp K)$.

(3) Since $p \notin K, q:=\widetilde{K}(p) \neq p$ and $(p, q) \in P^{(2)}$ hence $(p \perp K)=\{\overline{p, q}\}$.

(5) is a consequence of (1) and Theorem 1.1.

A symmetric 2-structure of class (III) is called point symmetric if $|K \cap L|=1$ for any $K, L \in \mathfrak{K}$ with $K \perp L$. If $a, b \in P$ are two given points then a point $m$ is called midpoint of $a$ and $b$ if $\widetilde{m}(a)=b$. Let $a * b$ denote the set of all midpoints of $a$ and $b$.

In [5] we proved that any two parallel points $a$ and $b$ have exactly one midpoint (cf. [5, Theorem 3.15]).

In this paper we construct for any two points $x, y$ (not necessarily parallel) the (uniquely determined) midpoint $x * y$ (cf. Theorem 2.2). From this it follows that each symmetric 2-structure of class (III) is already point symmetric (cf. Theorem 2.4). 
Let $\widetilde{P}:=\{\widetilde{p} \mid p \in P\}$ and $\sim: P \rightarrow \widetilde{P} ; x \mapsto \widetilde{x}$. From Theorem 1.1 follows that $(P, \sim)$ is an invariant involution set and from Theorem 2.4 that $\widetilde{P}$ acts regularly on $P$ hence $(P, \sim)$ is a point reflection structure and by $[2$, page 33 6.1.(3)] we have:

Theorem 1.3. If $o \in P$ is fixed, $p^{\prime}:=o * p, p^{+}:=\widetilde{p^{\prime}} \circ \widetilde{o}$ for $p \in P$ and if we set for $a, b \in P, a+b:=a^{+}(b)$, then $(P,+)$ is a K-loop uniquely 2-divisible.

Moreover we have (cf. Theorem 4.1.(4) for statement (1))

Theorem 1.4. Let $a, b \in P$ and $K \in \mathfrak{K}$ then:

1. $\tilde{a} \circ \tilde{b a} \circ \tilde{b}=\tilde{a b}$

2. If $[a]_{1}=[o]_{1}$ and $[b]_{2}=[o]_{2}$ then $a+b=b+a$.

3. $\widetilde{K}$ is an involutory automorphism of the invariant involution set $(P, \sim)$ (in particular if $m:=a * b$ then $\widetilde{K}(m)=\widetilde{K}(a) * \widetilde{K}(b)$ ) and an antiautomorphism of $(P, \square)$.

4. If $o \in K$ then $\widetilde{K}$ is an involutory automorphism of the loop $(P,+)$ interchanging the "axises" $X:=[o]_{1}$ and $Y:=[o]_{2}$.

\section{Each Symmetric 2-Structure of Class (III) is Point Symmetric}

Lemma 2.1. Let $(a, b) \in P^{(2)}, c \in P$ and $\tau:=\widetilde{a} \circ \widetilde{b}$ then $[c]_{i} \neq[\tau(c)]_{i}$ for $i \in\{1,2\}$.

Proof. (1) Let $A:=(a \perp \overline{a, b}), B:=(b \perp \overline{a, b})$ and $K=\overline{a, b}$ then by Theo$\operatorname{rem} 1.2 \widetilde{a}=\widetilde{A} \circ \widetilde{K}, \widetilde{b}=\widetilde{K} \circ \widetilde{B}, \tau=\widetilde{A} \circ \widetilde{B}, \tau(K)=K$ and Fix $\tau=\operatorname{Fix}(\widetilde{A} \circ \widetilde{B})=$ $A \cap B=\emptyset$ (by Theorem 1.2.(4)). $K \cap[c]_{i}$ consists of exactly one point $k$. Assume $[c]_{i}=[\tau(c)]_{i}$ then $\tau(k)=\tau\left(K \cap[c]_{i}\right)=\tau(K) \cap \tau\left([c]_{i}\right)=K \cap[c]_{i}=k$, a contradiction to Fix $\tau=\emptyset$.

Theorem 2.2. Let $\Sigma:=\left(P, \mathfrak{G}_{1}, \mathfrak{G}_{2}, \mathfrak{K}\right)$ be a symmetric 2-structure of class (III) and let $(a, b) \in P^{(2)}$. Then:

1. $a$ and $b$ have exactly one midpoint.

2. If $m=a * b$ then $m a=a * b a$ and $a m=a * a b$ and moreover $\tilde{m}(a b)=b a$.

3. If $m_{1}=a * a b$ and $m_{2}=a b * b$ then $m_{2} m_{1}=a * b$.

4. For $x, y \in P$ we have the formulas:

$x * y=y * x,(x * y) x=x *(y x), y(x * y)=y *(y x)$

$x * y=(x y) *(y x)=(x * y x)(y * y x)$.

5. $[\widetilde{a b}(b)]_{1}=\left\{\widetilde{x}(b) \mid x \in[a]_{1}\right\},[\widetilde{b a}(b)]_{2}=\left\{\widetilde{x}(b) \mid x \in[a]_{2}\right\}$ and $[b * a]_{i}=$ $\left\{b * x \mid x \in[a]_{i}\right\}$.

Proof. By [5, Theorem 3.15.], each of the pairs of parallel points $a$ and $a b, a$ and $b a, b$ and $b a$, and $b$ and $a b$ has exactly one midpoint $m_{1}, m_{2}, m_{1}^{\prime}$ and $m_{2}^{\prime}$. If $C:=\overline{a, b}$ and $D:=\overline{a b, b a}$ then by the proof of [5, Theorem 3.15.], $\widetilde{C}\left(m_{1}\right)=m_{2}$, 
$\widetilde{C}\left(m_{1}^{\prime}\right)=m_{2}^{\prime}$ and $\widetilde{D}\left(m_{1}\right)=m_{2}^{\prime}, \widetilde{D}\left(m_{2}\right)=m_{1}^{\prime}$. Thus $m_{2} m_{1}, m_{2}^{\prime} m_{1}^{\prime} \in C$ and $m_{2}^{\prime} m_{1}, m_{2} m_{1}^{\prime} \in D$. We consider the map $\tau:=\widetilde{m_{1}^{\prime}} \circ \widetilde{m_{1}}$ and obtain: $\tau\left([a]_{2}\right)=\widetilde{m_{1}^{\prime}}\left([a b]_{2}\right)=\widetilde{m_{1}^{\prime}}\left([b]_{2}\right)=[b a]_{2}=[a]_{2}$.

Since $(a, b) \in P^{(2)}$ we have $\left[m_{1}\right]_{1}=[a]_{1} \neq[b]_{1}=\left[m_{1}^{\prime}\right]_{1}$. Assume $\left[m_{1}\right]_{2} \neq$ $\left[m_{1}^{\prime}\right]_{2}$ then $\left(m_{1}, m_{1}^{\prime}\right) \in P^{(2)}$ and we get a contradiction with Lemma 2.1 since $\tau\left([a]_{2}\right)=[a]_{2}$. Therefore $\left[m_{1}\right]_{2}=\left[m_{1}^{\prime}\right]_{2}$ and in the same way, $\left[m_{2}\right]_{1}=\left[m_{2}^{\prime}\right]_{1}$. This gives us $m:=m_{2} m_{1}=m_{2} m_{1}^{\prime}=m_{2}^{\prime} m_{1}=m_{2}^{\prime} m_{1}^{\prime} \in C \cap D$, i.e. $m$ is the midpoint of $a$ and $b$ and by [5, Theorem 3.14.(2)] $m$ is unique. Moreover $\widetilde{m}(a b)=\widetilde{m}\left([a]_{1} \cap[b]_{2}\right)=[\widetilde{m}(a)]_{1} \cap[\widetilde{m}(b)]_{2}=[b]_{1} \cap[a]_{2}=b a$. Hence (1), (2) and (3) are proved. (4) and (5) are consequences of the previous items.

Corollary 2.3. Let $K \in \mathfrak{K}, a, b \in K, a \neq b$ and $i \in\{1,2\}$. Then:

1. If $L=(a \perp K),\{c\}=[b]_{i} \cap L$ and if $m$ is the midpoint of $b, c$ then $[m]_{3-i}=$ $[a]_{3-i}$.

2. If $m=b a$ or $m=a b$ and $c=\widetilde{m}(b)$ then $K \perp \overline{a, c}$.

Proof. (1) Let $b^{\prime}:=\widetilde{a}(b)$ and $i=1$. Then $c=b b^{\prime}$ and $L=\overline{b b^{\prime}, b^{\prime} b}$. Hence $m=b a$, by Theorem 2.2. i.e. $[m]_{2}=[a]_{2}$.

(2) We consider the case $m=b a$. Let $L=(a \perp K),\left\{c^{\prime}\right\}=[b]_{1} \cap L$ and $m^{\prime}$ is the midpoint of $b, c^{\prime}$. Then, by (1), $m^{\prime}=b a=m$ hence $L=\overline{a, c}$.

Theorem 2.4. Each symmetric 2-structure of class (III) is point symmetric.

Proof. Let $(A, B) \in \mathfrak{K}^{2 \perp}$ and $a \in A \backslash B$. By $A \perp B, b:=\widetilde{B}(a) \in A$ hence $A=\overline{a, b}, B=\overline{a b, b a}$ and by Theorem 2.2 there is exactly one midpoint $m$ of $a$ and $b$ and also of $a b$ and $b a$. Therefore $\widetilde{m}(A)=A, \widetilde{m}(B)=B$ and so $m \in A \cap B$. Thus $(A, B) \in \mathfrak{K}_{1}^{2 \perp}$.

Corollary 2.5. Let $\left(P, \mathfrak{G}_{1}, \mathfrak{G}_{2}, \mathfrak{K}\right)$ be a symmetric 2 -structure of class (III). If $(a, b) \in P^{(2)}, C:=\overline{a, b}, D=\overline{a b, b a}$ and $m:=a * b$ then $\{m\}=C \cap D=(a b \perp$ $C) \cap C=(a \perp D) \cap D, \widetilde{C}(a b)=b a$ and $\widetilde{D}(a)=b$.

\section{Reflections in Generators}

Let $\Sigma:=\left(P, \mathfrak{G}_{1}, \mathfrak{G}_{2}, \mathfrak{K}\right)$ be a point symmetric 2 -structure. For each $a \in P$ we have the 1-projection and the 2-projection

$\Pi_{a 1}: P \rightarrow[a]_{2} ; x \mapsto x a$ and $\Pi_{a 2}: P \rightarrow[a]_{1} ; x \mapsto a x$

and the generator reflections

$\widetilde{[a]_{1}}: P \rightarrow P ; x \mapsto \widetilde{a x}(x)$ and $\widetilde{[a]_{2}}: P \rightarrow P ; x \mapsto \widetilde{x a}(x)$

which are involutory permutations of $P$ fixing exactly the elements of the generators $[a]_{1}$ and $[a]_{2}$, respectively. By definition, $\widetilde{[a]_{1}}$ is a 2-map and from Theorem 2.2(5) follows that $\widetilde{[a]_{1}}$ takes any 1-generator into a 1-generator, 
hence $\widetilde{[a]_{1}}$ is an automorphisms of $\left(P, \mathfrak{G}_{1}, \mathfrak{G}_{2}\right) . \widetilde{[a]_{2}}$ is an automorphism too and a 1-map. Now let $K \in \mathfrak{K}$ and $\{p\}=K \cap[a]_{1}$ then, by Corollary 2.3., $\widetilde{[a]_{1}}(K)=(p \perp K)$. Hence $\widetilde{[a]_{1}}$ is also an automorphism of $(P, \mathfrak{K})$ and together with [5, Theorems 3.7 and 3.8.] we have the result:

Theorem 3.1. Let $\Sigma:=\left(P, \mathfrak{G}_{1}, \mathfrak{G}_{2}, \mathfrak{K}\right)$ be a point symmetric 2-structure and let $a \in P, K \in \mathfrak{K}$ and $i \in\{1,2\}$. Then:

1. If $\{p\}=K \cap[a]_{i}$ then $\widetilde{[a]_{i}}(K)=(p \perp K)$ hence each reflection in a generator is involutory and contained in $\Gamma^{+}(\mathfrak{K})$, more precisely, $\widetilde{[a]_{i}} \in \Gamma_{3-i}(\mathfrak{K})$ and we have $\widetilde{[a]_{i}}(K) \perp K$.

2. $\forall a \in P: \widetilde{[a]_{1}} \circ \widetilde{[a]_{2}}=\widetilde{[a]_{2}} \circ \widetilde{[a]_{1}}=\widetilde{a}$.

3. Let $A, B \in \mathfrak{K}$ with $A \perp B$ and $\{p\}=A \cap B$ then $\widetilde{[p]_{i}}(A)=B$.

4. Let $A, B \in \mathfrak{K}$ with $A \perp B$ and $\{p\}=A \cap B$ and let $\alpha \in \Gamma_{i}(\mathfrak{K})$ with $\alpha(A)=B$ then $\alpha=\widetilde{[p]_{3-i}}$.

5. If $A \in \mathfrak{K},\{p\}=A \cap[a]_{1},\{q\}=A \cap[a]_{2}, B:=(p \perp A)$ and $C:=(q \perp A)$ then $\widetilde{B A} \circ \widetilde{A}=\widetilde{A} \circ \widetilde{A B}=\widetilde{[a]_{1}}$ and $\widetilde{A C} \circ \widetilde{A}=\widetilde{A} \circ \widetilde{C A}=\widetilde{[a]_{2}}$.

6. If $A, B \in \mathfrak{K}$ with $A \perp B$ and $\{c\}:=A \cap B$ then $\widetilde{A B} \in \Gamma^{-}(\mathfrak{K})$ and $\widetilde{A B} \circ \widetilde{A B}=$ $\widetilde{A} \circ \widetilde{B}=\widetilde{c}$ is the reflection in the point $c$.

7. If $[a]_{i}=[b]_{i}$ then $(\widetilde{a} \circ \widetilde{b}) \in \Gamma_{i}(\mathfrak{K})$.

8. Any involution of $\Gamma_{i}(\mathfrak{K})$ is the reflection in a generator of $\mathfrak{G}_{3-i}$.

Proof. (2) Follows from [5, Theorem 3.8.(3)].

(3) Since $B=(p \perp A)$ we have by $(1), \widetilde{[p]_{i}}(A)=B$.

(4) By (1), $\widetilde{[p]_{3-i}} \in \Gamma_{i}(\mathfrak{K})$ and $\widetilde{[p]_{3-i}}(A)=B$. Therefore $\widetilde{[p]_{3-i}}$ and also $\alpha$ induce the perspectivity $[A \stackrel{i}{\longrightarrow} B]$ or in other words, $\widetilde{[p]_{3-i}}$ and $\alpha$ are extensions of the perspectivity $[A \stackrel{i}{\longrightarrow} B]$ and so by [3, Theorem 2.4.], $\alpha=\widetilde{[p]_{3-i}}$.

(5) Follows from (3) and [3, Theorem 2.9.(4),(5)].

(6) Follows from (2) and (5).

(7) By (2), $\widetilde{a}=\sigma_{1} \circ \sigma_{2}$ and $\tilde{b}=\sigma_{2} \circ \sigma_{3}$ where $\sigma_{1}, \sigma_{2}$ and $\sigma_{3}$ are reflections in the generators $[a]_{3-i},[a]_{i}=[b]_{i}$ and $[b]_{3-i}$ respectively. Hence $\widetilde{a} \circ \widetilde{b}=$ $\sigma_{1} \circ \sigma_{3} \in \Gamma_{i}(\mathfrak{K})$. (8) Let $\alpha \in \Gamma_{i}(\mathfrak{K})$ be an involution, let $x \in P \backslash$ Fix $\alpha, y:=\alpha(x)$ and $m:=x * y$. Then $\alpha(m)=m$ and since $\alpha \in \Gamma_{i}(\mathfrak{K}), \alpha$ fixes all elements of the generator $[m]_{3-i}$. Thus $\alpha=\widetilde{[m]_{3-i}}$.

Let $\mathfrak{B}:=\mathfrak{G}_{1} \cup \mathfrak{G}_{2} \cup \mathfrak{K}$ be the set of all blocks. Then $(P, \mathfrak{B})$ is an incidence space, i.e. any two distinct points $a, b \in P$ can be joined by exactly one block of $\mathfrak{B}$ also denoted by $\overline{a, b}$. An automorphism $\delta \in \Gamma(\mathfrak{K})$ is called a

latation if for $x \in P$ with $\delta(x) \neq x: \delta(\overline{x, \delta(x)})=\overline{x, \delta(x)}$ dilatation if for $B \in \mathfrak{B}: \delta(B)=B$ or $\delta(B) \cap B=\emptyset$ 
translation if $\delta$ is the identity or if $\delta$ is a latation and a dilatation without fixed points.

If $\sigma$ is an involutory automorphism of $(P, \mathfrak{B})$ then $\sigma$ is a latation.

Corollary 3.2. If $[a]_{i}=[b]_{i}$ then $\tau ;=\widetilde{a} \circ \widetilde{b}$ is an $i$-map and a translation.

Proof. By the proof of Theorem $3.1(7), \tau=\widetilde{a} \circ \widetilde{b}=\widetilde{[a]_{3-i}} \circ \widetilde{[b]_{3-i}} \in \Gamma_{i}(\mathfrak{K})$ hence $\tau$ is an i-map and so also a latation. If $K \in \mathfrak{K}$ then by Theorem 3.1 (1),

$$
K \perp \widetilde{[b]_{3-i}}(K) \perp \widetilde{[a]_{3-i}} \circ \widetilde{[b]_{3-i}}(K)=\widetilde{a} \circ \widetilde{b}(K) .
$$

Hence by Theorem 1.2(4), $\widetilde{a} \circ \widetilde{b}(K)=K$ or $\widetilde{a} \circ \widetilde{b}(K) \cap K=\emptyset$, i.e. $\tau$ is a translation.

\section{Midpoint Configurations}

For two subsets $X, Y \subseteq P$ let $X * Y:=\{x * y \mid x \in X, y \in Y\}$ and for $a \in P$ let $a * X:=\{a\} * X$.

Theorem 4.1. Let $a, b, c, p \in P, X, Y \in \mathfrak{G}_{i}, i \in\{1,2\}$ and $A \in \mathfrak{K}$ with $p \notin A$. Then:

1. $p * X, X * Y \in \mathfrak{G}_{i}$ hence if $x \in X, y \in Y$ then $p * X=[p * x]_{i}$ and $X * Y=[x * y]_{i}$.

2. $\widetilde{p}(X)=Y \Leftrightarrow p \in X * Y$.

3. If $[a]_{i}=[b]_{i}$ then $[\widetilde{a}(c)]_{i}=[\widetilde{b}(c)]_{i}$ and so $\tau:=\widetilde{a} \circ \widetilde{b}$ is an i-map.

4. $\widetilde{a} \circ \widetilde{a b}=\widetilde{b a} \circ \widetilde{b}$ is a 1-map and $\widetilde{a} \circ \widetilde{b a}=\widetilde{a b} \circ \widetilde{b}$ is a 2-map.

5. If $a_{i}:=[p]_{i} \cap A$ and $\left\{p^{\prime}\right\}:=(p \perp A) \cap A$ then $p * p^{\prime} \in \overline{p * a_{1}, p * a_{2}}$.

6. Let $[a]_{i}=[b]_{i}, a \neq b$ and $x \in[a * b]_{3-i} \backslash\{a * b\}$ then $\overline{a, x} \perp \overline{b, x}$.

Proof. (1) Let for instance $X \in \mathfrak{G}_{1},\left\{p^{\prime}\right\}:=[p]_{2} \cap X$ and $x \in X$ then $x p=p^{\prime}$ and so by Theorem 2.2.(2), $(p * x) p=p *(x p)=p * p^{\prime}$ implying $p * X=[p * x]_{1}=$ $\left[p * p^{\prime}\right]_{1} \in \mathfrak{G}_{1}$. If $p, q \in Y$ hence $[p]_{1}=[q]_{1}=Y$ and if $\left\{q^{\prime}\right\}:=[q]_{2} \cap X$ then $\left[p^{\prime}\right]_{1}=\left[q^{\prime}\right]_{1}=X$ and $p * X=\left[p * p^{\prime}\right]_{1}, q * X=\left[q * q^{\prime}\right]_{1}$. Again by Theorem 2.2.(2), $\left[p * p^{\prime}\right]_{1}=\left[p * q^{\prime}\right]_{1}=\left[q * q^{\prime}\right]_{1}$. Therefore $Y * X=\left[p * p^{\prime}\right]_{1} \in \mathfrak{G}_{1}$.

(2) " $\Rightarrow$ " If $x \in X$ then $y:=\widetilde{p}(x) \in \widetilde{p}(X)=Y$, hence $p=x * y \in X * Y$. "६" Let $x \in X, y \in Y$ with $p=x * y$, i.e. $\widetilde{p}(x)=y$. Then $\widetilde{p}(X)=\widetilde{p}\left([x]_{i}\right)=$ $[\widetilde{p}(x)]_{i}=[y]_{i}=Y$.

(3) By (1) and assumption we have $b \in[a]_{i}=[c]_{i} *[\widetilde{a}(c)]_{i}$ and so by (2), $[\widetilde{b}(c)]_{i}=\widetilde{b}\left([c]_{i}\right)=\widetilde{a}\left([c]_{i}\right)=[\widetilde{a}(c)]_{i}$.

(4) Since $[a b]_{1}=[a]_{1}$ and $[b a]_{2}=[a]_{2}$, by (3), $\widetilde{a} \circ \widetilde{a b}$ and $\tilde{b a} \circ \tilde{b}$ are 1-maps, and $\widetilde{a} \circ \widetilde{b a}$ and $\widetilde{a b} \circ \widetilde{b}$ are 2-maps. Therefore for $x \in P$ we have $\widetilde{a} \circ \widetilde{a b}\left([x]_{1}\right)=\widetilde{b a} \circ$ $\widetilde{b}\left([x]_{1}\right)=[x]_{1}$ and $\widetilde{a b}\left([x]_{2}\right)=\widetilde{b}\left([x]_{2}\right), \widetilde{a}\left([x]_{2}\right)=\widetilde{b a}\left([x]_{2}\right)$ implying $\widetilde{a} \circ \widetilde{a b}\left([x]_{2}\right)=$ $\widetilde{b a} \circ \widetilde{b}\left([x]_{2}\right)$. Together with $x=[x]_{1} \cap[x]_{2}$, we obtain $\widetilde{a} \circ \widetilde{a b}(x)=\widetilde{b a} \circ \widetilde{b}(x)$. Thus $\widetilde{a} \circ \widetilde{a b}=\widetilde{b a} \circ \widetilde{b}$ and in the same way $\widetilde{a} \circ \widetilde{b a}=\widetilde{a b} \circ \widetilde{b}$. 
(5) Since $p=a_{1} a_{2}$ and so $\widetilde{A}(p)=a_{2} a_{1}$ we have by Corollary $2.5 p^{\prime}=a_{2} *$ $a_{1}$ and then by Theorem 2.2.(4), $p^{\prime}=\left(a_{2} * a_{1} a_{2}\right) \square\left(a_{1} * a_{1} a_{2}\right)=\left(a_{2} * p\right) \square\left(a_{1} * p\right)$ and so $p^{\prime} * p=\left(a_{2} * p\right) *\left(a_{1} * p\right)$ hence $p * p^{\prime} \in \overline{p * a_{1}, p * a_{2}}$.

(6) Follows from Theorem 3.1.(1) since $a=\left[\widetilde{a * b]_{3-i}}(b)\right.$.

Theorem 4.2. If $\Sigma:=\left(P, \mathfrak{G}_{1}, \mathfrak{G}_{2}, \mathfrak{K}\right)$ is a point symmetric 2-structure and if we consider on $P$ the binary operations $a \square b:=a b=[a]_{1} \cap[b]_{2}, a * b(=$ midpoint of $a$ and $b)$ and $a \circ b:=\widetilde{a}(b)$ for $a, b \in P$ then $(P, \square, *, \circ)$ satisfies the following rules (let $a, b, c \in P$ ):

1. $(P, \square),(P, *)$ and $(P, \circ)$ are idempotent, i.e. $a=a a=a * a=a \circ a$.

2. $(P, \square)$ is associative moreover $a(b c)=(a b) c=a c$.

3. $(P, *)$ is commutative.

4. $(a * b) \circ a=b$ and $a *(b \circ a)=b$ hence $(P, \circ)$ and $(P, *)$ are quasigroups.

5. $a(b * c)=(a b) *(a c),(a * b) c=(a c) *(b c), a(b \circ c)=(a b) \circ(a c),(a \circ b) c=$ $(a c) \circ(b c)$ and $a \circ(b * c)=(a \circ b) *(a \circ c)$.

For each $B \in \mathfrak{B}, B$ is a substructure of $(P, *, \circ)$ and $(B, \circ),(B, *)$ are idempotent quasigroups, if $A, B \in \mathfrak{B}$ then $(A, *, \circ)$ and $(B, *, \circ)$ are isomorphic.

Proof. (5) By Theorem 2.2.(4) we have:

$(*) a(a * b)=a *(a b)$

and so also by using $(2),(a b) *(a c)=((a c) b) *(a c)=(a c)(b * a c)=(a b)(b *$ $(a c))=a(b(b *(a c)))=a(b *(b c))=a b(b * c)=a(b * c)$. From $b=c *(b \circ c)$ and $a(b * c)=(a b) *(a c)$ it follows that $a b=(a c) *(a(b \circ c))$. Hence $(a b) \circ(a c)=$ $a((b \circ c)$ By Theorem 1.4.(3), $\widetilde{a}(b * c)=\widetilde{a}(b) * \widetilde{a}(c)$, i.e. $a \circ(b * c)=(a \circ b) *(a \circ c)$. Now let $A, B \in \mathfrak{B}$. If $B \in \mathfrak{K}$ and $a \in P$ then $\pi_{a, 1}: B \rightarrow[a]_{1} ; b \mapsto a b$ and $\pi_{a, 2}: B \rightarrow[a]_{2} ; b \mapsto b a$ are bijections and for $b, c \in B$ we have by (5): $\pi_{a, 1}(b * c)=a(b * c)=(a b) *(a c)=\pi_{a, 1}(b) * \pi_{a, 1}(c), \pi_{a, 2}(b * c)=(b * c) a=$ $\pi_{a, 2}(b) * \pi_{a, 2}(c), \pi_{a, 1}(b \circ c)=a(b \circ c)=(a b) \circ(a c)=\pi_{a, 1}(b) \circ \pi_{a, 1}(c)$ and $\pi_{a, 2}(b \circ c)=(b \circ c) a=\pi_{a, 2}(b) \circ \pi_{a, 2}(c)$ hence $\pi_{a, 1}$ and $\pi_{a, 2}$ are isomorphisms from $(B, *, \circ)$ onto $\left([a]_{1}, *, \circ\right)$ and onto $\left([a]_{2}, *, \circ\right)$. Consequently $(A, *, \circ)$ and $(B, *, \circ)$ are isomorphic.

\section{Products of i-maps and the Corresponding K-loop}

For $i \in\{1,2\}$ let $T_{i}:=\left\{\widetilde{a} \circ \widetilde{b} \mid a, b \in P\right.$ with $\left.[a]_{i}=[b]_{i}\right\}$. Then by Theorem 4.1.(3), $T_{i}$ is a set of i-maps.

By $[5$, Theorem 3.16] and $[2, \mathbf{6 . 1}$.$] we have$

Theorem 5.1. Let $\Sigma:=\left(P, \mathfrak{G}_{1}, \mathfrak{G}_{2}, \mathfrak{K}\right)$ be a point symmetric 2 -structure, let $o \in P$ be fixed, for $p \in P$ let $p^{\prime}:=o * p$ and $p^{+}:=\widetilde{p^{\prime}} \circ \widetilde{o}$. If we set for $a, b \in P, a+b:=a^{+}(b)$, then $(P,+)$ is a K-loop uniquely 2-divisible. 
$(P,+)$ is the loop derivation in the point o (cf. [2, Definition 1]). For any two points $o_{1}, o_{2}$ the reflection in the midpoint $o_{1} * o_{2}$ establishes an isomorphism between the loops derived in the points $o_{1}$ and $o_{2}$. For the following let $\Sigma$ be a point symmetric 2 -structure, let $o \in P$ be fixed, let $(P,+)$ be the K-loop derived in $o$ and let $X:=[o]_{1}, Y:=[o]_{2}$.

Theorem 5.2. Let $\widetilde{X} \circ \widetilde{X}:=\{\widetilde{a} \circ \widetilde{b} \mid a, b \in X\}, \widetilde{Y} \circ \widetilde{Y}:=\{\widetilde{a} \circ \widetilde{b} \mid a, b \in Y\}, X^{+}:=$ $\left\{x^{+} \mid x \in X\right\}$ and $Y^{+}:=\left\{y^{+} \mid y \in Y\right\}$ then:

1. $X^{+} \subseteq T_{1}=\widetilde{X} \circ \widetilde{X}$ and $Y^{+} \subseteq T_{2}=\widetilde{Y} \circ \widetilde{Y}$, more precisely, if $a, b \in P$ with $[a]_{1}=[b]_{1}$ then $\widetilde{a} \circ \widetilde{b}=\widetilde{o a} \circ \widetilde{o b}$.

2. $X$ and $Y$ are subloops of $(P,+)$ and $\forall p \in P \exists_{1}(x, y) \in X \times Y: p=x+y$.

3. $\forall(x, y) \in X \times Y x+y=y+x$ even $x^{+} \circ y^{+}=y^{+} \circ x^{+}$.

4. If $o \in E \in \mathfrak{K}$ then the loops $(E,+),(X,+),(Y,+)$, are isomorphic.

Proof. (1) By the definitions of $X, Y, X^{+}$and $Y^{+}$and by Theorem 4.1.(3) we have $X^{+} \subseteq \widetilde{X} \circ \widetilde{X} \subseteq T_{1}$ and $Y^{+} \subseteq \widetilde{Y} \circ \widetilde{Y} \subseteq T_{2}$. Now let $a, b \in P$ with $[a]_{1}=[b]_{1}$ then $o a, o b \in X, b=a \square(o b)$ and $o a=(o b) \square a$ hence by Theorem 4.1.(4), $\widetilde{a} \circ \widetilde{b}=\widetilde{a} \circ \widetilde{a \square(o b)}=\widetilde{(o b) \square a} \circ \widetilde{o b}=\widetilde{o a} \circ \widetilde{o b} \in \widetilde{X} \circ \widetilde{X}$ hence $T_{1}=\tilde{X} \circ \widetilde{X}$.

(2), (3) Let $a \in X$. Since $o \in X, a^{\prime}=o * a \in X$ and so $\widetilde{o}(X)=\widetilde{a^{\prime}}(X)=X$ implying $a+X=a^{+}(X)=X$. Moreover if $a, b \in X$ then $x_{1}:=\widetilde{o} \circ \widetilde{a^{\prime}}(b) \in$ $\widetilde{o} \circ \widetilde{a^{\prime}}(X)=X$ is the solution of the equation $a+x=b$ cotained in $X$ and since the midpoint $m:=\widetilde{o}(a) * b \in X$ is contained in $X$ also $x_{2}:=\widetilde{m}(o) \in X$ thus $x_{2}+a=\widetilde{x_{2}^{\prime}} \circ \widetilde{o}(a)=\widetilde{m} \circ \widetilde{o}(a)=b$ and so the solution $x_{2}$ of $x+a=b$ is contained in $X$. Therefore $X$ and $Y$ are subloops of $(P,+)$.

If we set $\{x\}:=X \cap[p]_{2},\{y\}:=Y \cap[p]_{1}$ then $o=x y, p=y x$ and by Theorem 4.1, $[p * y]_{2}=Y *[p]_{2}=[o * x]_{2}$ imlying $p * y=(o * x) \square y=x^{\prime} y$ hence by (1), $x^{+}=\widetilde{x^{\prime}} \circ \widetilde{o}=\widetilde{p * y} \circ \widetilde{y}$ and we have $x+y=x^{+}(y)=\widetilde{p * y} \circ \widetilde{y}(y)=\widetilde{p * y}(y)=p$ and since $x^{\prime} \square y^{\prime}=o$ we have by Theorem 4.1.(4), $\widetilde{x^{\prime}} \circ \widetilde{o} \circ \widetilde{y^{\prime}}=\widetilde{y^{\prime} x^{\prime}}$ implying $\widetilde{x^{\prime}} \circ \widetilde{o} \circ \widetilde{y^{\prime}}=\widetilde{y^{\prime}} \circ \widetilde{o} \circ \widetilde{x^{\prime}}$ hence $x^{+} \circ y^{+}=y^{+} \circ x^{+}$. If there are $a \in X, b \in Y$ with $p=a+b$ then $p=x+y=\widetilde{x^{\prime}} \circ \widetilde{o}\left(\widetilde{y^{\prime}}(o)\right)=\widetilde{y^{\prime} x^{\prime}}(o)=\widetilde{b^{\prime} a^{\prime}}(o)$ hence $p^{\prime}=o * p=y^{\prime} x^{\prime}=b^{\prime} a^{\prime}$ and so $y^{\prime}=b^{\prime}$ and $x^{\prime}=a^{\prime}$, i.e. $y=\widetilde{y^{\prime}}(o)=\widetilde{b^{\prime}}(o)=b$ and in the same way, $x=a$.

(4) We have $a+b=(a * o) \circ(o \circ b)$. Hence, by the proof of the last statement of Theorem 4.2, $\pi_{o, 1}$ and $\pi_{o, 2}$ are isomorphisms from $(E,+)$ onto $(X,+)$ and onto $(Y,+)$.

Open Access. This article is distributed under the terms of the Creative Commons Attribution Noncommercial License which permits any noncommercial use, distribution, and reproduction in any medium, provided the original author(s) and source are credited. 


\section{References}

[1] Karzel, H.: Zusammenhänge zwischen Fastbereichen, scharf zweifach transitiven Permutationsgruppen und 2-Strukturen mit Rechtecksaxiom. Abh. Math. Sem. Univ. Hamburg 32, 191-206 (1968)

[2] Karzel, H.: Loops related to geometric structures. Quasigroups Relat. Syst. 15, 47-76 (2007)

[3] Karzel, H., Kosiorek, J., Matraś, A.: Properties of auto-and antiautomorphisms of maximal chain structures and their relations to i-perspectivities. Result. Math. 50, 81-92 (2007)

[4] Karzel, H., Kosiorek, J., Matraś, A.: Automorphisms of symmetric and double symmetric chain structures. Result. Math. 55, 401-416 (2009)

[5] Karzel, H., Kosiorek, J., Matraś, A.: Symmetric 2-structures, a classification, Result.Math., submited

[6] Karzel, H.: Inzidenzgruppen (Vorlesungsausarbeitung von I. Pieper und K. Sörensen) Hamburg (1965)

Helmut Karzel

Zentrum Mathematik

T.U. München

80290 München

Germany

e-mail: karzel@ma.tum.de

Jarosław Kosiorek and Andrzej Matraś

Faculty of Mathematics and Computer Science

University of Warmia and Mazury in Olsztyn

Żołnierska 14

10-561 Olsztyn

Poland

e-mail: kosiorek@matman.uwm.edu.pl;

matras@uwm.edu.pl

Received: October 14, 2009.

Revised: October 15, 2010.

Accepted: December 1, 2010. 